\title{
A PINCHING THEOREM FOR CUSPS OF NEGATIVELY CURVED MANIFOLDS WITH FINITE VOLUME
}

\author{
MASAHIKO KANAI \\ (Communicated by Jonathan M. Rosenberg) \\ Dedicated to Professor Shingo Murakami on his 60th birthday
}

\begin{abstract}
We give a new proof of the following theorem of M. Gromov: For a noncompact complete riemannian manifold $M$ of negative curvature with finite volume, each cusp of $M$ is diffeomorphic to $N \times[0, \infty)$ with $N$ being a compact flat space form provided that the sectional curvature of $M$ satisfies the pinching condition $-4<-\Lambda^{2} \leq K \leq-1$.
\end{abstract}

\section{INTRODUCTION}

One of the main problems in differential geometry that has been attracting us is to reveal relationships between curvature and topology of riemannian manifolds. A celebrated success in this direction is the pinching theorem in positive curvature due to M. Berger and W. Klingenberg (cf. [CE], [Sa]), which claims that a closed simply connected riemannian manifold with sectional curvature $1 \leq K<4$ is homeomorphic to the sphere. It had led us to the pinching problem in negative curvature as well-Is a closed riemannian manifold with sectional curvature $-4<K \leq-1$ homeomorphic to a space of constant negative curvature? However Gromov and Thurston [GT] gave counter-examples to the problem in any dimension greater than three. Although the original pinching problem fails in negative curvature, it still remains possible that pinching curvature of negatively curved manifolds involves topological obstructions. In fact, Hamenstädt $[\mathrm{H}]$ recently proved a beautiful theorem which especially suggests that if $M$ is a closed locally symmetric space of negative curvature other than the spaces of constant curvature then $M$ never carries a metric of curvature $-4<K \leq-1$ (cf. Ville [V] and Pansu [P]).

Meanwhile noncompact manifolds of negative curvature with finite volume as well as compact ones have been studied extensively. In particular the purpose of the present paper is to indicate a topological obstruction to pinching curvature of a noncompact negatively curved manifold with finite volume. To be more precise, suppose that $M$ is a noncompact complete riemannian manifold of

Received by the editors February 22, 1988 and, in revised form, April 25, 1988.

1980 Mathematics Subject Classification (1985 Revision). Primary 53C20; Secondary 53C22. 
finite volume whose sectional curvature $K$ satisfies the pinching condition

$$
-\Lambda^{2} \leq K \leq-1
$$

Then it is known (cf. [G1], [E], [Sc]) that each end (or cusp) of $M$ is topologically of the form

$$
N \times[0, \infty),
$$

where $N$ is a closed infranil manifold. Note here that in case $M$ is locally symmetric, $N$ is homeomorphic to a flat space form if and only if $M$ is of constant curvature. The theorem below means that the pinching condition (1) with $\Lambda<2$ implies that the ends of $M$ are topologically the same with those of a space of constant curvature.

Theorem. Suppose that $M$ is a noncompact complete riemannian manifold of finite volume whose sectional curvature satisfies the pinching condition

$$
-4<-\Lambda^{2} \leq K \leq-1
$$

Then each cusp of $M$ is diffeomorphic to $N \times[0, \infty)$ with $N$ being a closed flat space form.

The theorem was first announced by Gromov [G3], and a detailed proof based on Gromov's almost flat theory [G2] was given by Buser and Karcher [BK]. In this note we will give a new proof of Gromov's theorem.

\section{PROOF OF THE THEOREM}

We give a proof of the theorem in the rest of the paper, which proceeds as follows. First in $\S 2.1$ we will show that the metric of the manifold in the theorem can be deformed to another one which is more convenient for our purpose. Then we turn to the review of the geometric description of the cusps $(\S 2.2)$, and prove a few inequalities from the curvature assumption $(\S 2.3)$. The most crucial part in the proof of the theorem is to find a torsion-free affine connection $D$ on the infranil manifold $N$ in (2). This will be done in $\S 2.4$. In addition, by showing that $D$ is flat $(\S 2.5)$, and that $N$ has a riemannian metric which is parallel with respect to $D(\S 2.6)$, the proof of the theorem will be completed. The construction of the connection $D$ is closely related to the preceding work [K1], [K2] of the author.

2.1. To begin with we should refer to the work of W.-X. Shi on the deformation of riemannian metrics. Suppose in general that $g$ is a complete riemannian metric on a differentiable manifold $M$ whose sectional curvature $K_{g}$ satisfies the pinching condition $A \leq K_{g} \leq B$. Then Shi's theorem [Sh] claims that for any $\delta>0$ one can deform the metric $g$ to another metric $g^{\prime}$ of $M$ that satisfies the following conditions: (i) $g^{\prime}$ is quasi-isometric to $g$ in the sense that there is a constant such that const ${ }^{-1} \cdot g(X, X) \leq g^{\prime}(X, X) \leq$ const $\cdot g(X, X)$ for all $X \in T M$; (ii) The sectional curvature $K_{g^{\prime}}$ of $g^{\prime}$ satisfies the pinching 
condition $A-\delta \leq K_{g^{\prime}} \leq B$; (iii) The covariant derivative of the curvature tensor $R_{g^{\prime}}$ of $g^{\prime}$ with respect to the riemannian connection $\nabla_{g^{\prime}}$ of $g^{\prime}$ is uniformly bounded; i.e., $\left|\nabla_{g^{\prime}} R_{g^{\prime}}\right| \leq$ const .

In particular by applying this to the metric of the manifold $M$ in the theorem and by replacing the metric of $M$ by the deformed one, we may assume further, in the theorem, that the covariant derivative $\nabla R$ of the curvature tensor of $M$ is uniformly bounded. In other words it is enough for us to prove the theorem under the additional condition that $|\nabla R| \leq$ const. This subordinate condition will be utilized in $\S 2.3$.

2.2. We now review the geometric description (2) of cusps of $M$ (see Gromov [G1], Eberlein [E] and Schroeder [Sc] for details). As before, let $M$ be a noncompact complete riemannian manifold of finite volume whose sectional curvature satisfies the pinching condition (1) for some $\Lambda \geq 1$, and take an end, say $e$, of $M$. We can always find a length-minimizing geodesic ray $r=r(t)$ $(0 \leq t<\infty)$ of $M$ diverging to $e$. For simplicity, assume that $r$ is of unit speed. Then for the Busemann function $b$ relative to $r$ that is defined by

$$
b(x)=\lim _{t \rightarrow \infty}\{t-d(x, r(t))\} \quad(x \in M),
$$

there is a constant $T$ satisfying the following conditions: (i) $b$ is $C^{2}$-differentiable on $b^{-1}(T, \infty)$; (ii) $|\operatorname{grad} b| \equiv 1$ on $b^{-1}(T, \infty)$; (iii) $b^{-1}(t)$ is compact for any $t \in(T, \infty)$. By reparametrizing $r$ if necessary, we may assume $T<0$. Since $b$ is a Morse function on $b^{-1}(T, \infty)$, the cusp $b^{-1}[0, \infty)$ is diffeomorphic to the product space $N \times[0, \infty)$ with $N=b^{-1}(0)$.

2.3. Next we give a few estimates for the gradient flow of the Busemann function $b$. Put $N_{t}=b^{-1}(t)$ for $t \geq 0$. Then the gradient flow of $b$ gives rise to a $C^{1}$ diffeomorphism $\varphi_{a}: N_{t} \rightarrow N_{t+a}$ for any $t, a \geq 0$, and under the assumption (1), it satisfies the following $C^{1}$-estimate which is an immediate consequence of the standard comparison argument (cf. Heintze-Im Hof [HI]):

$$
e^{-\Lambda a} \cdot|X| \leq\left|d \varphi_{a} X\right| \leq e^{-a} \cdot|X|, \quad X \in T N_{t} .
$$

To obtain a $C^{2}$-estimate, assume further that $\Lambda<2$ in (1) and that $|\nabla R| \leq$ const for the covariant derivative of the curvature tensor of $M$ (cf. $\S 2.1$ ). Under these conditions, Green [Gr] proved that the Busemann function $b$ is $C^{3}$ on $b^{-1}[0, \infty)$. In particular the diffeomorphism $\varphi_{a}: N_{t} \rightarrow N_{t+a}$ is $C^{2}$ differentiable. Now denote the riemannian connection of the hypersurface $N_{t}$ of $M$ by $\nabla_{t}$. Then the pull-back $\varphi_{a}^{*} \nabla_{t+a}$ is a torsion-free $C^{0}$ affine connection of $N_{t}$, and $\nabla_{t}^{2} \varphi_{a}=\varphi_{a}^{*} \nabla_{t+a}-\nabla_{t}$ is a continuous $(1,2)$-tensor field on $N_{t}$. It is easy to see that $(d / d a) \nabla_{t}^{2} \varphi_{a}=\varphi_{a}^{*}\left(\left.(d / d \alpha)\right|_{\alpha=0} \nabla_{t}^{2} \varphi_{\alpha r}\right)$. In the right hand side, $\varphi_{a}^{*}: T^{*} N_{t+a} \otimes T^{*} N_{t+a} \otimes T N_{t+a} \rightarrow T^{*} N_{t} \otimes T^{*} N_{t} \otimes T N_{t}$ is induced from $d \varphi_{a}: T N_{t} \rightarrow T N_{t+a}$ that satisfies the inequalities (3), and therefore we have $\left|\varphi_{a}^{*}\right| \leq e^{-(2-\Lambda) a}$. In addition we can follow arguments of Green [Gr] once 
again to show that $\left|(d / d \alpha) \nabla_{t}^{2} \varphi_{\alpha}\right|_{\alpha=0} \leq$ const on $N_{t}$, where the constant is independent of $t \geq 0$. Hence we obtain

$$
\left|\frac{d}{d a} \nabla_{t}^{2} \varphi_{a}\right| \leq \text { const } \cdot e^{-(2-\Lambda) a} .
$$

Since $\Lambda<2$, this immediately implies a uniform estimate

$$
\left|\nabla_{t}^{2} \varphi_{a}\right| \leq \mathrm{const}
$$

on $N_{t}$ with a constant independent of $t, a \geq 0$.

2.4. It is now possible to show that the family of the continuous affine connections $\varphi_{t}^{*} \nabla_{t}$ on $N=N_{0}$ converges to an affine connection $D$ of $N$ in the $C^{0}$-topology as $t$ goes to infinity. In fact, for $a, t \geq 0$, we have

$$
\left|\varphi_{t+a}^{*} \nabla_{t+a}-\varphi_{t}^{*} \nabla_{t}\right|=\left|\varphi_{t}^{*}\left(\nabla_{t}^{2} \varphi_{a}\right)\right| \leq\left|\varphi_{t}^{*}\right| \cdot\left|\nabla_{t}^{2} \varphi_{a}\right| \leq \text { const } \cdot e^{-(2-\Lambda) t}
$$

by (4) and the fact that $\left|\varphi_{t}^{*}\right| \leq e^{-(2-\Lambda) t}$. Since $\Lambda<2$, this inequality actually guarantees that $\varphi_{t}^{*} \nabla_{t}$ converges uniformly to a $C^{0}$ affine connection $D$ of $N$ as $t \rightarrow \infty$. Of course $D$ is torsion-free since so are $\varphi_{t}^{*} \nabla_{t}$ 's.

2.5. The next purpose is to exhibit the flatness of the affine connection $D$ on $N=N_{0}$ we have just obtained. Note here that $D$ is only known to be continuous, and the curvature tensor of $D$ is not defined in general. However the $D$-parallel translation along a smooth curve in $N$ is always well defined, since the equation of the parallel translation is, in this case, a first order linear ordinary differential equation with continuous coefficients. What we are going to see here is that the $D$-parallel translation depends only on the homotopy class of the curve. Namely, let $c=c(s) \quad(0 \leq s \leq 1)$ be a homotopically trivial loop in $N=N_{0}$ with base point $x=c(0)=c(1) \in N$, and for each tangent vector $X \in T_{x} N$, denote the $D$-parallel translation of $X$ along $c$ by $X(s) \in T_{c(s)} N$ $(0 \leq s \leq 1)$. Then we should prove that $X(0)=X(1)$. Let $X_{t}(s)$ be the $\varphi_{t}^{*} \nabla_{t}$-parallel translation of $X$ along $c$. Then $X_{t}(s)$ converges to $X(s)$ as $t$ goes to $\infty$, since $\varphi_{t}^{*} \nabla_{t}$ tends to $D$ uniformly. Thus it is enough to show

$$
\left|X_{t}(0)-X_{t}(1)\right| \rightarrow 0 \quad \text { as } t \rightarrow \infty \text {. }
$$

To prove this, let $c_{t}=\varphi_{t} \circ \mathrm{c}$ be the translation of the loop $c$ in $N$ by the diffeomorphism $\varphi_{t}: N \rightarrow N_{t}$, and $Y_{t}(s)$ the $\nabla_{t}$-parallel translation of $Y_{t}=$ $d \varphi_{t} X$ along $c_{t}$. Then it is obvious that

$$
Y_{t}(s)=d \varphi_{t} X_{t}(s)
$$

Now we can show that

$$
\left|Y_{t}(1)-Y_{t}(0)\right| \leq \text { const } \cdot e^{-t} \cdot\left|Y_{t}(0)\right|
$$

for sufficiently large $t$ in the following way. First lift both the loop $c_{t}$ in $N_{t} \subset M$ and the parallel translation $Y_{t}(s)$ along $c_{t}$ to the universal covering $\widetilde{M}$ of $M$. Then we obtain a loop $\tilde{c}_{t}=\tilde{c}_{t}(s)$ in a lift $\tilde{N}_{t} \subset \widetilde{M}$ of $N_{t}$, and 
a parallel translation $\tilde{Y}_{t}(s)$ along $\tilde{c}_{t}$. Notice here that the equation of the parallel translation along $\tilde{c}_{t}(s)$ to which $\tilde{Y}_{t}(s)$ is a solution is a first order linear ordinary differential equation with continuous coefficients, and the coefficients involve only the Christoffel symbols of $\widetilde{M}$, the second fundamental form of the horosphere $\tilde{N}_{t}$, and the velocity vector $\tilde{c}_{t}^{\prime}$ of the loop $\tilde{c}_{t}$. Since the loop $\tilde{c}_{t}$ contracts exponentially as $t \rightarrow \infty, \tilde{c}_{t}$ is enclosed in a certain geodesic ball $U=U_{t}$ in $\widetilde{M}$ of radius 1 whenever $t$ is large enough. Besides, the coefficients of the metric tensor as well as the Christoffel symbols of $\widetilde{M}$ relative to normal coordinates are uniformly bounded on the unit ball $U$, since $\widetilde{M}$ satisfies $|R|$, $|\nabla R| \leq$ const (cf. Aubin [A, p. 153]). On the other hand, by (1), the second fundamental form of the horosphere $\tilde{N}_{t}$ is also bounded: This again follows from Rauch's comparison theorem (cf. [HI]). Finally, by (3), the velocity vector $\tilde{c}_{t}^{\prime}$ satisfies $\left|\tilde{c}_{t}^{\prime}\right| \leq$ const $\cdot e^{-t}$. In consequence the coefficients of the equation of the parallel translation along $\tilde{c}_{t}$ expressed in terms of normal coordinates on $U$ are dominated by const $\cdot e^{-t}$. Thus, by applying a standard inequality for linear ordinary differential equations to the solution $\widetilde{Y}_{t}(s)$ of the equation of the parallel transformation along $\tilde{c}_{t}(s)$, we have $\left|\tilde{Y}_{t}(1)-\tilde{Y}_{t}(0)\right| \leq$ const $_{1} \cdot \exp (-t) \cdot$ $\exp \left\{\right.$ const $\left._{2} \cdot \exp (-t)\right\} \cdot\left|\tilde{Y}_{t}(0)\right|$, and this immediately implies $(7)$.

Combining (6), (7) and (3), we obtain

$$
\left|X_{t}(1)-X_{t}(0)\right| \leq \text { const } \cdot e^{-(2-\Lambda) t} \cdot|X|
$$

for any sufficiently large $t$. This proves the desired convergence (5), and the continuous affine connection $D$ on $N$ turns out to be flat in the sense that the $D$-parallel translation is determined only by the homotopy class of a path of the translation.

2.6. To complete the proof of the theorem it is now sufficient to show that $N=N_{0}$ carries a $C^{1}$ riemannian metric $h$ such that $D h \equiv 0$. Let $g_{t}$ be the induced riemannian metric of the hypersurface $N_{t}$ of $M$. Then the pull-back $\varphi_{t}^{*} g_{t}$ of $g_{t}$ by the $C^{2}$ diffeomorphism $\varphi_{t}: N \rightarrow N_{t}$ is a $C^{1}$ riemannian metric of $N$. Now fix a point $x$ in $N$, and normalize $\varphi_{t}^{*} g_{t}$ so that its norm at $x$ relative to the metric $g_{0}$ is equal to 1 . Denoting the normalized metric by $h_{t}$, we obviously have $\left(\varphi_{t}^{*} \nabla_{t}\right) h_{t} \equiv 0$. Hence for any $y \in N, h_{t}(y)$ is the $\varphi_{t}^{*} \nabla_{t^{-}}$ parallel translation of $h_{t}(x)$ along a curve combining $x$ and $y$. Recall now that $\varphi_{t}^{*} \nabla_{t}$ converges uniformly to $D$. On the other hand, by the normalization $\left|h_{t}(x)\right|_{g_{0}} \equiv 1$, we can find a diverging sequence $\left\{t_{k}\right\}$ so that $h_{t}(x)$ tends to a positive-semidefinite symmetric bilinear form $h^{(0)}(x)$ of $T_{x} N$ as $t=t_{k} \rightarrow$ $\infty$. Hence $h_{t}(y)$ also converges to $h^{(0)}(y)$ that is the $D$-parallel translation of $h^{(0)}(x)$. The resulting positive-semidefinite symmetric $(0,2)$-tensor field $h^{(0)}$ on $N$ is clearly parallel with respect to $D$.

In case $h^{(0)}$ is nondegenerate, the proof has been already finished. Otherwise we can proceed as follows. Let $E^{(1)}$ be the null space of $h^{(0)}$, which is a $C^{1}$ 
subbundle of $T N$ closed under the covariant derivative by $D$, and $g_{t}^{(1)}$ be the induced riemannian metric of the subbundle $E_{t}^{(1)}=d \varphi_{t} E^{(1)}$ of $T N_{t}$. We can then apply the argument above again, and show that $E^{(1)}$ possesses a $D$-parallel positive-semidefinite symmetric bilinear form $h^{(1)}$. Repeating this procedure, we obtain sequences of bundles $T N=E^{(0)} \supset E^{(1)} \supset \cdots$, and of $D$-parallel positive-semidefinite symmetric bilinear forms $h^{(0)}, h^{(1)}, \ldots$ on them such that each $E^{(k)}$ is the null space of $h^{(k-1)}$. Obviously $h=h^{(0)}+h^{(1)}+\cdots$ is a desired riemannian metric of $N$ that is parallel with respect to the torsion-free "flat" $C^{0}$ affine connection $D$.

In consequence, $N$ turns out to be diffeomorphic to a flat space form, and the proof of the theorem is completed.

\section{ACKNOWLEDGMENT}

The author wishes to thank Michael Gromov, Wan-Xiong Shi and Hajime Tsuji for their valuable suggestions. In particular conversation with $\mathrm{H}$. Tsuji and his result in $[\mathrm{T}]$ partially initiated the present work.

\section{REFERENCES}

[A] T. Aubin, Espaces de Sobolev sur les variétés riemanniennes, Bull. Sci. Math. (2) 100 (1976), 149-173.

[BK] P. Buser and H. Karcher, Gromov's almost flat manifolds, Astérisque, no. 81, Société Mathématique de France, 1981.

[CE] J. Cheeger and D. G. Ebin, Comparison theorems in riemannian geometry, North-Holland, Amsterdam, 1975.

[E] P. Eberlein, Lattices in spaces of nonpositive curvature, Ann. of Math. 111 (1980), 435-476.

[Gr] L. W. Green, The generalized geodesic flow, Duke Math. J. 41 (1974), 115-126; Correction, ibid. 42 (1975), p. 381.

[G1] M. Gromov, Manifolds of negative curvature, J. Diff. Geom. 13 (1978), 223-230.

[G2] __, Almost flat manifolds, J. Diff. Geom. 13 (1978), 231-241.

[G3] _ Synthetic geometry in riemannian manifolds, in "Proceedings of The International Congress of Mathematics, Helsinki, 1978," pp. 415-419.

[GT] M. Gromov and W. Thurston, Pinching constants for hyperbolic manifolds, Invent. Math. 89 (1987), 1-12.

[H] U. Hamenstädt, in preparation.

[HI] E. Heintze and H.-C. Im Hof, Geometry of horospheres, J. Diff. Geom. 12 (1977), 481-491.

[K1] M. Kanai, Geodesic flows of negatively curved manifolds with smooth stable and unstable foliations, Ergod. Th. Dynam. Sys. 8 (1988), 215-239.

[K2] __ Tensorial ergodicity of geodesic flows in "Geometry and Analysis on Manifolds," Springer Lectures Notes in Math., no. 1339, 1988, pp. 142-157.

[P] P. Pansu, Quasiconformal mappings and manifolds of negative curvature, in "Curvature and Topology of Riemannian Manifolds," Springer Lecture Notes in Math., no. 1201, 1986, pp. 212-229.

[Sa] T. Sakai, Comparison and finiteness theorems in riemannian geometry, in "Geometry of Geodesics and Related Topics," Advanced Studies in Pure Math., vol. 3, North-Holland, Amsterdam, 1984, pp. 125-181. 
[Sc] V. Schroeder, Finite volume and fundamental group on manifolds of negative curvature, J. Diff. Geom. 20 (1984), 175-183.

[Sh] W.-X. Shi, Deforming the metric on complete riemannian manifolds, J. Diff. Geom. 30 (1989), 223-301.

[T] H. Tsuji, Complete negatively curved Kähler surfaces of finite volume (to appear).

[V] M. Ville, Sur les variétés riemanniennes 1/4-pincées de dimension 4 et de courbure négative, C. R. Acad. Sci. Paris, Sér. I 300 (1985), 397-400.

Department of Mathematics, Keio University, Köhoku-Ku, Yokohama 223, Japan 